\title{
Zebrafish as an in Vivo Screen for Early Black Cranberry Proanthocyanidin Biomolecular Activity
}

\author{
Nicholas J. Macedo ${ }^{1}$, Catherine C. Neto ${ }^{2}$, Anne M. Liberty ${ }^{2}$, Tracie L. Ferreira ${ }^{1}$ \\ ${ }^{1}$ Department of Bioengineering, The University of Massachusetts Dartmouth, North Dartmouth, USA \\ ${ }^{2}$ Department of Chemistry/Biochemistry, The University of Massachusetts Dartmouth, North Dartmouth, USA \\ Email: tferreira@umassd.edu
}

Received 11 January 2014; revised 10 February 2014; accepted 8 March 2014

Copyright (C) 2014 by authors and Scientific Research Publishing Inc.

This work is licensed under the Creative Commons Attribution International License (CC BY). http://creativecommons.org/licenses/by/4.0/

\section{Open Access}

\section{Abstract}

Antioxidants have been widely studied in various naturally occurring substances as a bioavailable cancer prevention treatment. Proanthocyanidins (PACs), which are abundant polyphenols in Early Black (EB) Cranberry (Vaccinium macrocarpon), are readily available and we have shown their anticancer activity in several cancer cell lines. This work focused on the activity of these compounds when incorporated into the zebrafish (Danio rerio) system. We began investigating the in vivo effect of these phytochemicals, the protective role of several other cranberry compounds, and the metabolic activity of the vertebrate model organism. Proanthocyanidin fractions were separated from fresh EB Cranberry fruit by chromatography on Sephadex LH-20 in order to acquire a workable stock solution in DMSO. Various concentrations of proanthocyanidins in solution were tested against fish ranging in age from 1-cell stage to adult level of growth. Acridine orange apoptosis indicator dye was incorporated into the treatment protocol, and it was observed that irregular epithelial cell death was occurring in treated embryos but not in the control group. Further apoptosis assays were carried out utilizing Dihydroethidium (DHE) superoxide sensitive dye in the treatment protocol. Fluorescing red nuclei were visible along the outer surface of the epithelium cell layer; an indication of superoxide release within cells leading to the nicking of DNA within the nucleus. It was also possible to screen for superoxide release in PACs treated CCD-CO18 and HT-29 cells using confocal microscopy and cell apoptosis was investigated by trypan blue cytotoxicity assay; cell apoptosis results were statistically significant as confirmed by ANOVA analysis. Results indicate that the phytochemicals may induce apoptosis in rapidly dividing cells.

\section{Keywords}

Zebrafish; In Vivo; Proanthocyanidins

How to cite this paper: Macedo, N.J., Neto, C.C., Liberty, A.M. and Ferreira, T.L. (2014) Zebrafish as an in Vivo Screen for Early Black Cranberry Proanthocyanidin Biomolecular Activity. American Journal of Molecular Biology, 4, 37-48. 


\section{Introduction}

The Cranberry (Vaccinium macrocarpon) has gained the attention of researchers investigating human infectious disease because of the fruit's strong, natural antioxidant-rich content. The berries are considered to be a promising resource for carcinoma prevention since they are an abundant source of polyphenols. The long-term benefits of cranberry consumption have become increasingly relevant to research in heart disease treatment, in that daily consumption of concentrated cranberry extract or whole organic cranberry juice has the potential to reduce the risk of heart attack and stroke. In a study geared towards coronary heart disease research, investigators showed that by allowing affected subjects to consume double-strength cranberry juice with a 835 mg total polyphenol content resulted in a reduction of carotid femoral wave velocity and in turn decreased arterial stiffness which was observed as soon as 4 hours after subjects had consumed a $480 \mathrm{~mL}$ portion of the cranberry juice [1]. Experimentation in the utilization of cranberry for combating microbial infections suggests that it is effective against human pathogenic bacteria, for example, urinary tract infections caused by harboring Escherichia coli (E. coli) in the colon. One study demonstrated that by exposing a wide variety of bacteria in culture to cranberry-based phenols that there was an overall inhibition of cell growth including both gram-negative and gram-positive bacteria [2]. Another study showed that at a concentration of cranberry phenols in solution of $100 \mu \mathrm{g} / \mathrm{mL}$ it was possible to significantly inhibit the growth of a wide range of microbes. In that study, it was determined that the most hydrophobic fractions found in the cranberry fruit resulted in the highest degree of antimicrobial activity and those fractions were characterized to be enriched with proanthocyanidins [3]. The phytochemical makeup in cranberries includes several classes of flavonoids such as flavonols, anthocyanins, and proanthocyanidins which are classified further as phenolic constituents and the antioxidant properties of these polyphenolic compounds in cranberry fruit may contribute to the observed anti-cancer activities of cranberry extracts [4]. The complex structure of proanthocyanidin molecules may hold the key to understanding why the extracts exhibit anti-cancer properties [5] [6]. Extractable proanthocyanidin subfractions from whole fruit have been observed to be composed of 4 - 7 epicatechin units with A-type linkages between units [7] as confirmed by mass spectrometry (MALDI-TOF MS) characterization and other studies suggest that A-type PACs may exhibit heightened cytotoxic activity as compared to B-type linkages [8]. One study has shown that NCI-H460 esophageal and lung cancer cells treated with cranberry PACs experienced increased cytochrome C presence in the cytoplasm which then resulted in an incremental increase in apoptosis over a 72-hour period with an overall 1.3-fold production since time of treatment [9]. The incorporation of cranberry products in an individual's daily nutritional regimen could bring about a variety of health benefits as demonstrated by these studies with a potentially broad scope of protection.

One of the first steps in advancing a pharmaceutical drug or a medication to the human usage stage is the determination of dosage level as a function of how the body may metabolize the drug by employing in vivo assays. The zebrafish is a vertebrate that has proven to be a practical candidate for human disease analysis and prevention due to the high level of similarity between the two genomes [10]-[13]. Zebrafish embryos can be bred frequently and develop fully functioning organ systems over the course of 48 hours post fertilization (hpf) [14] [15], giving researchers the ability to carry out experimental procedures within a short period of time. Studies have shown that zebrafish can spontaneously develop any type of tumor [16], thus continue to be a valuable resource in discovering methods for treating human carcinoma; therefore, zebrafish may be exposed to anti-cancer medications as an initial trial procedure to gain data on the vitally important toxicity factors including dosage dependency and reactivity with metabolizing organs such as the liver and blood filtrating organs of the kidney. Variants of anti-cancer pleurocidin peptides have been experimentally proven to induce apoptosis in human leukemia (HL60) cells and be highly toxic on the cellular level at low concentrations, resulting in organelle deactivation including mitochondrial shut-down [17]. While being a highly toxic defense mechanism to cultured cancer cells, the peptides were ultimately as toxic to zebrafish embryos resulting in yolk sac rupture and disassociation of animal pole cells [17]. It is of the utmost importance to investigate these topics in drug development, since not all biological systems have the same capacity to break down toxins or resist damage caused by highly reactive medications.

\section{Materials and Methods}

\subsection{Proanthocyanidin Fractions}

Proanthocyanidin A2 dimers (A2 PAC) were obtained from Indofine Chemical Company, Inc (Hillsborough, NJ, 
USA).

EB Cranberry crude extract was prepared by exhaustive extraction of whole cranberry fruit with 40:40:19:1 acetone/methanol/water/formic acid mixture, concentrating in vacuum, and lyophilization. The extract was desugared by ion exchange chromatography on Diaion HP-20 and eluted with methanol, then separated on a Sephadex LH-20 column as described previously [18]. Proanthocyanidins with DP $<10$ (PACs) were eluted using 70:30 Acetone $/ \mathrm{H}_{2} \mathrm{O}$ after elution of other flavonoids with methanol/ $\mathrm{H}_{2} \mathrm{O}$, lyophilized, and stored at $-20^{\circ} \mathrm{C}$ until use.

A similar method was used to separate small proanthocyanidins with DP $<6$ (smPACs) from the flavonol fraction obtained from methanol/ $\mathrm{H}_{2} \mathrm{O}$ elution.

Purity of both samples were confirmed by HPLC-DAD on a Waters Millenium system, using a $4.6 \times 250$ Xterra C8 column using 2\% $\mathrm{HOAc} / \mathrm{H}_{2} \mathrm{O}$ as solvent " $\mathrm{A}$ " and $2 \% \mathrm{HOAc} / \mathrm{MeOH}$ as solvent "B" with gradient elution at $0.8 \mathrm{~mL} / \mathrm{min}$ from $100 \%$ A to $100 \%$ B from 0 - 50 minutes, followed by isocratic elution with $100 \%$ "B" from 60 - 90 minutes, monitoring at $280 \mathrm{~nm}$. The characteristic proanthocyanidin elution profile in comparison to previously reported data [7] [18] confirmed the presence of PACs (Figure 1).

\subsection{Cell Lines}

Non-cancerous human colon (CCD-CO18) and cancerous human colon (HT-29) cells were obtained from ATCC and cultured at $37^{\circ} \mathrm{C}$ and $5.0 \% \mathrm{CO}_{2}$. CCD-CO18 cells were cultured in EMEM-F12 medium with $10 \%$ fetal bovine serum (FBS) while HT-29 cells were cultured in McCoy's 5A modified media also containing 10\% FBS. Cells were allowed to reach approximately $85 \%$ confluence in T75 cell culture flasks before passaging into a final volume of $2 \mathrm{~mL}$ in $35 \mathrm{~mm}$ glass bottom microwell petri dishes from MatTek Corporation to allow for confocal imaging 24 hours after passaging.

\subsection{Treatment of Zebrafish with Proanthocyanidins}

Zebrafish larvae of various ages (1-cell, 4-cell, high, 24 hpf, 36 hpf, 48 hpf, 3 day, 5 day, 7 day, 2 month old) were initially used to assay the effect of PACs on development and survival. Micro-well culture plates were utilized to dilute the extract in distilled water to concentrations of $0.625,1.25,2.5,5,10,20,30,40,50$, and 100 $\mu \mathrm{g} / \mathrm{mL}$ from the initial proanthocyanidin stocks. Adult zebrafish (approximately 2 month old) were also treated with both eluted samples of proanthocyanidins as previously mentioned in distilled water, but at a larger final volume of $30 \mathrm{~mL}$ and only at 10,50 , and $100 \mu \mathrm{g} / \mathrm{mL}$ concentration (data not shown). The assayed zebrafish were allowed to swim in PACs dissolved in solution at $28.5^{\circ} \mathrm{C}$.

\subsection{Gene Expression Analysis}

The PACs stock solution was prepared at a concentration of $100 \mu \mathrm{g} / \mathrm{mL}$ in distilled water and was used to investigate specific organ function upon extract exposure. The distilled water control group was tested against the PACs treated group using 48 hpf zebrafish in a 12- well plate with final volumes at $2 \mathrm{~mL}$ with 30 fish occupying each well. Control and treated groups were homogenized at 45 minutes, 1.5 hours, and 2.25 hours post-exposure to the PACs treatment in $250 \mu \mathrm{L}$ of TRIzol ${ }^{\circledR}$ reagent by electronic mortar and pestle homogenization method [22]. Following this homogenization procedure, TRIzol ${ }^{\circledR}$ protocol was used to isolate mRNA from each sample and then reverse transcribed by Oligo $(\mathrm{dT})_{20}$ primer to yield cDNA using the SuperScript ${ }^{\mathrm{TM}}$ III First-Strand Synthesis kit (Invitrogen Carlsbad, CA). Liver function was assayed via CYP3A65 expression, kidney filtration activity via $A H R 2$ expression, and inflammation via COX2A expression using the gene specific forward and reverse primers as summarized in Table $1 ; \beta$-actin was used as a loading control. The PCR reactions were analyzed by gel electrophoresis and imaged under UV trans-illumination with AlphaEase ${ }^{\circledR}$ FC software from Alpha Innotech.

\subsection{Detection of Apoptotic Cells along the Zebrafish Epithelium}

Acridine orange apoptosis indicator dye was diluted from $300 \mathrm{X} \rightarrow 1 \mathrm{X}$ concentration and added to wells during the treatment assays. PACs at a final concentration of $100 \mu \mathrm{g} / \mathrm{mL}$ in $2 \mathrm{~mL}$ final volume was achieved in the 12well plate. Zebrafish at $48 \mathrm{hpf}$ were transferred to each of 8 wells and were allowed to swim at $28.5^{\circ} \mathrm{C}$. Imaging with the Olympus DP70 camera under fluorescent microscopy for green fluorescing proteins of treated and non- 


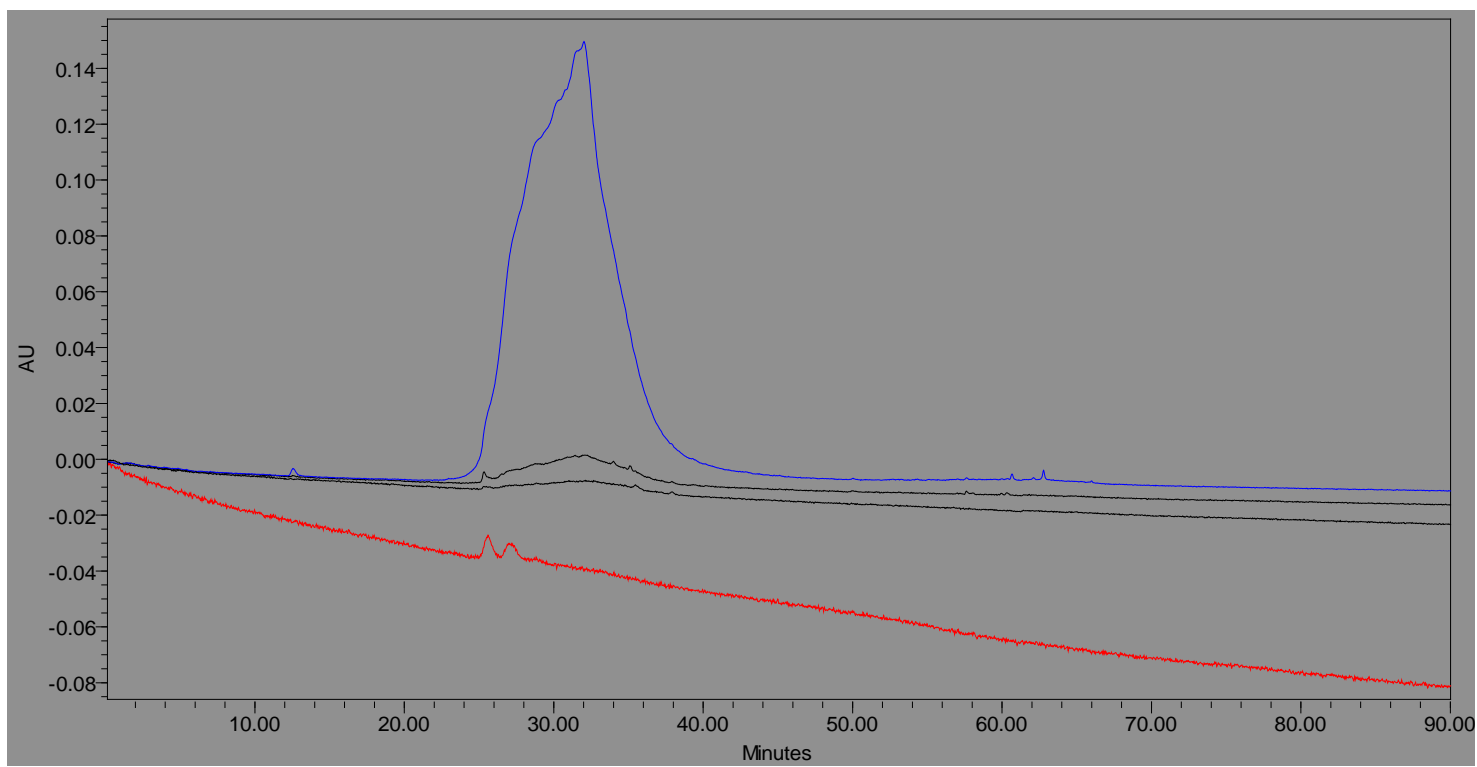

(a)

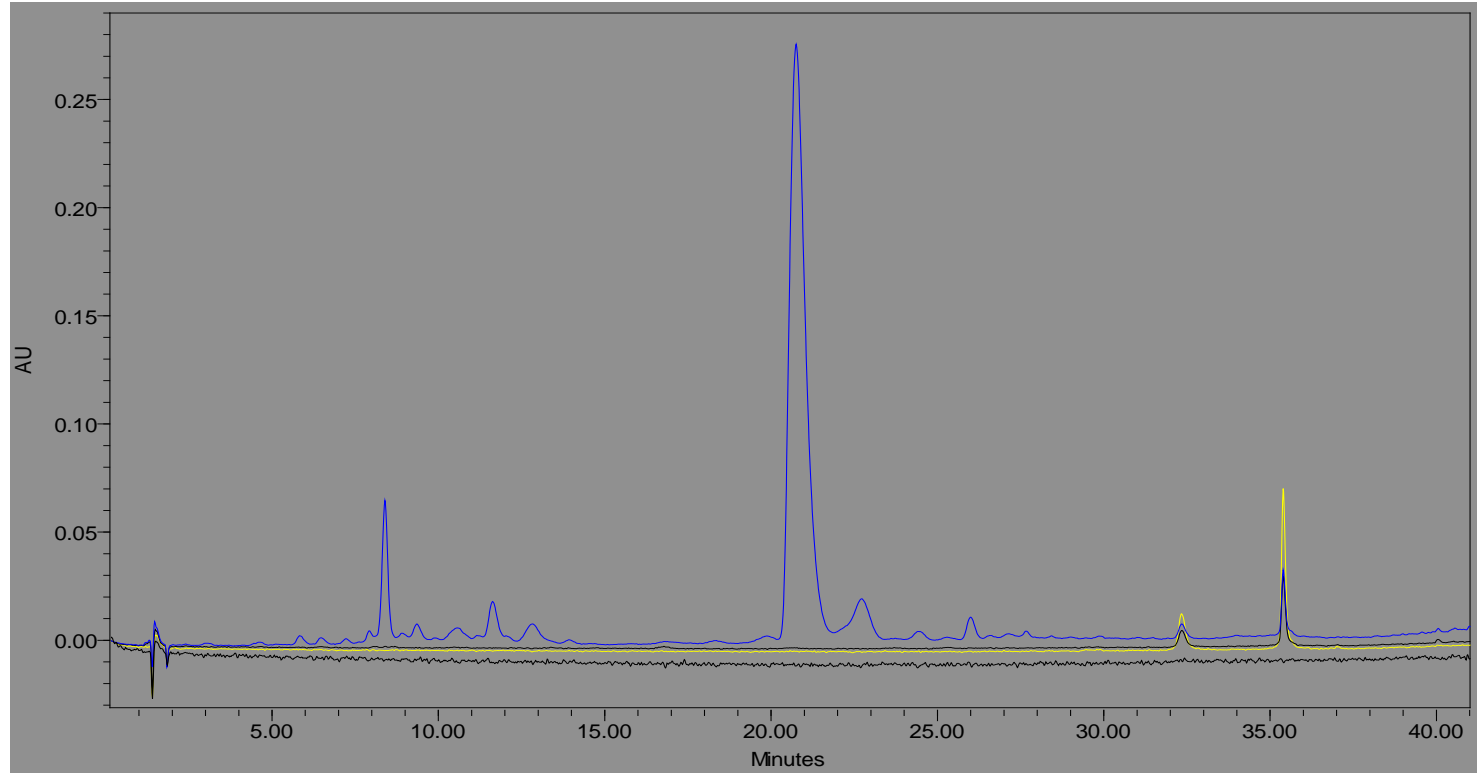

(b)

Figure 1. Chromatograms of eluted proanthocyanidin samples; (Top Panel), PACs at 280 nm; (Bottom Panel), smPACs at $280 \mathrm{~nm}$.

treated zebrafish took place at exposure times of 1 hour, 1 hour and 55 minutes, 2 hours and 35 minutes, and 5 hours. DHE superoxide sensitive dye was received from Invitrogen ${ }^{\mathrm{TM}}$ Molecular Probes ${ }^{\circledR}$ (Oregon, USA) as solid dihydroethidium (DHE), and solubilized in DMSO. The DHE stock was diluted to $3 \mu \mathrm{M}$ [7] in the treatment assays. The same timing protocol was followed exactly as for the acridine orange staining method to ensure uniformity in results in regards to the age of the zebrafish and the amount per well. Instead, imaging occurred under fluorescent microscopy for red fluorescing nicked- DNA of treated and non-treated zebrafish and at the same exposure times as previously mentioned.

\subsection{Cytotoxicity Assay}

Cells were incubated with $100 \mu \mathrm{g} / \mathrm{mL}$ PACs in microwell dishes for 5 hours with a negative control containing 
Table 1. Primers used for reverse transcription amplifications.

\begin{tabular}{ccc}
\hline Gene Name & Forward & Reverse \\
\hline$\beta$-Actin & 5'- GGAGAAGATCTGGCATCACACCTTCTAC - 3’ & 5' - TGGTCTCGTGGATACCGCAGATTCCAT - 3' \\
CYP3A65 & 5' - CTGTGCATCATGGACCAAAC - 3' & 5'- AGAGAGGGTTCAGCAGGTCA - 3' \\
AHR2 & 5' - CAGGAGGAAATGGATGGCTA - 3' & 5' - GGGATTGAGAGCGAAGTG - 3' \\
COX2A & 5' - AGGGCGTGTGTTTATCCAAG - 3' & 5' - ACCTGGACGTCCTTCATAAG - 3' \\
\hline
\end{tabular}

no treatment. Old media was removed and transferred to a sterile tube for use in dead cell counting while live cells were trypsinized before being transferred into sterile PBS and Trypan Blue $(0.4 \%$ w/v) followed by live and dead cell counting by hemocytometer reading and carried out in triplicate.

\subsection{Confocal Microscopy}

Cells were treated with $100 \mu \mathrm{g} / \mathrm{mL}$ PACs and stained with $3 \mu \mathrm{M}$ DHE in media while being imaged in real time under a characteristic reference dye programmed in the microscope system by the name of Ethidium Homodimer-1 for detection of a red fluorescent wavelength of $514 \mathrm{~nm}$ and confocal identification of superoxide presence in the cells at 1 hour, 1 hour and 55 minutes, 2 hours and 35 minutes, and 5 hours post-exposure to the treatment. Images were captured using Zen 2008 Software under a Zeiss LSM 710 laser scanning confocal microscope (Carl Zeiss, Thornwood, NY, USA). A 63X oil immersion objective was used.

\subsection{Statistical Methods}

One-way analysis of variance (ANOVA) was used to identify significant differences in apoptosis across multiple cell populations in response to PACs treatment. A standard significant level was used, and comparisons of the F statistic were made.

\section{Results \& Discussion}

\subsection{Phenotypic Effects of PACs Exposure}

The amount of time that the zebrafish could survive in the PACs treatment was concentration-dependent. We utilized $48 \mathrm{hpf}$ embryos for all assays since the large screen of various ages revealed no difference in embryo survival, and at $48 \mathrm{hpf}$ the liver and kidney are functioning. The fish survived for only 3.5 hours when exposed to either 2.5 or $5 \mu \mathrm{g} / \mathrm{mL}$ concentration, but could live for 5 hours at the $0.625 \mu \mathrm{g} / \mathrm{mL}$ concentration. At a concentration of $100 \mu \mathrm{g} / \mathrm{mL}$, the fish survived for a maximum of 2.5 hours at all other ages (Figure 2).

\subsection{Analysis of Metabolic Response to PACs}

By reverse transcribing the mRNA isolated from samples treated with PACs over the course of 2.25 hours, it was observed that gene function of the liver by expression of CYP3A65 was up regulated as kidney activity as assayed by $A H R 2$ expression increased in activity as compared to untreated controls during the 2.25 HR exposure time point (Figure 3), but revealed no clear explanation of the initial 45 min characteristic in both control and treated fish. Inflammatory control via COX2A regulatory gene was comparable in controls and treated fish as exposure to the treatment continued during the 45 minute treatment period, and continued to decrease in expression in treated fish at the 1.5 hours duration based on the control while the control groups served as a steady baseline in gene activity throughout all exposure time points (Figure 3). $\beta$-Actin was uniformly expressed in each sample throughout all conditions of treatment and control.

\subsection{Epithelial Apoptosis Visualization}

Epithelial apoptosis was observed in real time under fluorescent microscopy using acridine orange indicator, revealing disassociating cells along the epithelium of the $100 \mu \mathrm{g} / \mathrm{mL}$ PACs treated $48 \mathrm{hpf}$ zebrafish (Figure 4(d)) which was not observed in the distilled water control group (Figures 4(b) and (c)). The yolk sac of the embryos 


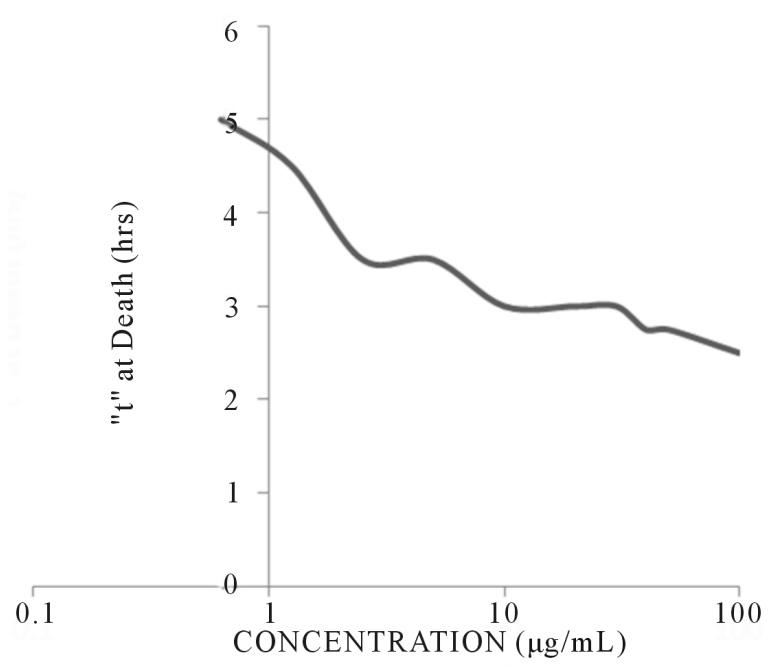

Figure 2. Viability of 48 hpf embryos upon PACs exposure (with DP < 10). Capser embryos were exposed to 0.625, $1.25,2.5,5,10,20,30,40,50$, and $100 \mu \mathrm{g} / \mathrm{mL}$ of PACs and survival was monitored. PACs concentrations affected the fish independent of age, and similar effects were seen across all ages with the same concentration.

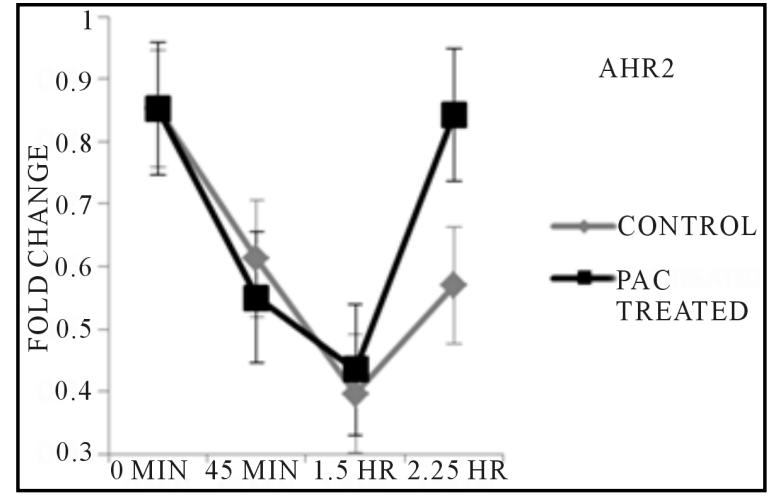

(a)

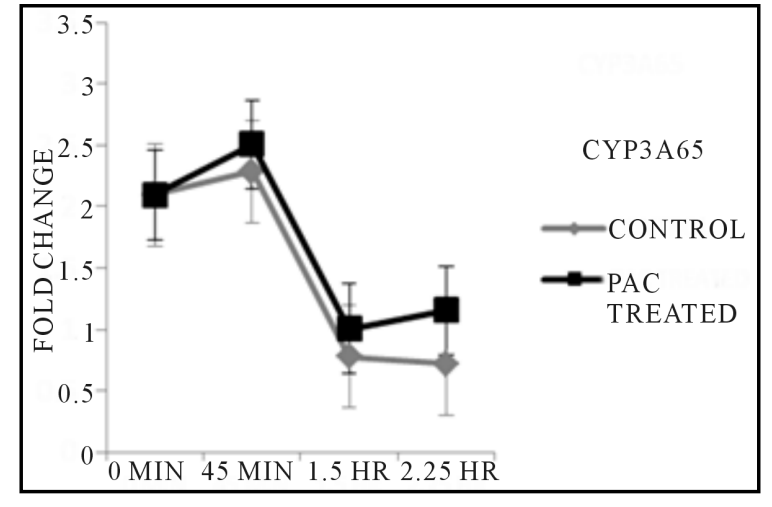

(b)

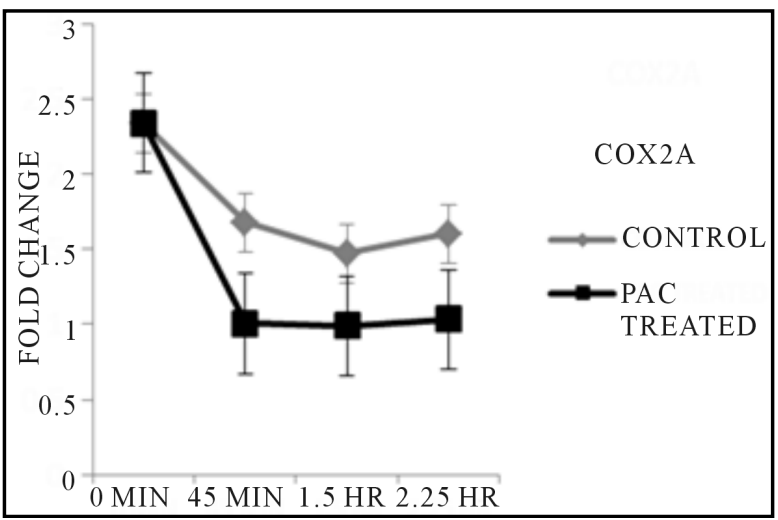

(b)

Figure 3. Results from PCR Reactions. CYP3A65 expression showed increased gene activity at the final exposure time point when compared to untreated control, while the expression of $A H R 2$ followed a similar trend in overall activity across later time points. Inflammatory response as regulated by $C O X 2 A$ expression was expressed at lower levels across all time points based on comparisons made with each untreated control group with corresponding exposure time. 
was the first area of the body to be affected by the treatment and was being damaged at as soon as 1 hour of exposure to the treatment (Figure 4(d)). Over the course of 5 hours, the reaction of the PACs with the zebrafish epithelial layer produced a heightened effect causing the integrity of the zebrafish tissue to diminish. It was observed that the PACs affected specific areas of tissue along the fish, which were localized around the olfactory epithelium and the urogenital pore region before the reaction began to affect the entire surface of the zebrafish epithelium layer (Figures 4(e) and (f)). In allowing different age groups to be exposed to the extract, no difference in cellular response to the phytochemical was observed; all ages were affected by the PACs by 1 hour of exposure at the earliest to approximately 2.5 hours at the time of severe system failure and overall death. Adult zebrafish were treated with $100 \mu \mathrm{g} / \mathrm{mL}$ of the extract and within 2.5 hours of exposure to the PACs, the fish was in critical condition and could no longer swim. Embryos at 4.5 and $6 \mathrm{hpf}$ were allowed to be exposed to variable concentrations of the extract at 10,20 , and $100 \mu \mathrm{g} / \mathrm{mL}$ with one treated group that had been dechorionated and one group that had not; both were also exposed to acridine orange. Over the course of 1 hour, cells of the 4.5 and 6 hpf dechorionated embryos had begun to show signs of cell apoptosis and disassociation as observed under fluorescence of incorporated acridine orange dye (data not shown). In contrast, the chorionated 4.5 and 6 hpf embryos experienced no apoptotic cellular behavior in treatment by displaying no uptake of the acridine orange indicator. By 2.5 hours of exposure, the 4.5 and 6 hpf embryo cells were completely disassociated from the yolk. In all cases, $\mathrm{pH}$ levels were tested digitally and there was no significant change from the initial $\mathrm{pH}$ of 7.0 in the treatment solution.

DHE staining in solution of 48 hpf PACs treated and non-treated controls revealed the presence of superoxides within the cell nuclei of the fish epithelial tissue cells as indicated by red fluorescing nuclei. The point that marked the beginning of significant reactions to the treatment was observed at 2 hours and 35 minutes in PACs treated fish, and it was possible to detect cell apoptosis by superoxide release along the entire length of the tail (Figure 5(j)) and localized cell death at the olfactory area (Figure 5(h)). At 5 hours of exposure to the treatment, smPACs treated fish had suffered the same degree of tissue damage as the PAC treated fish mainly in the area of the body around the yolk sac but also concentrated at areas around the tail (Figure 5(c)). Fish treated with the dimer proanthocyanidin A2 were not affected by the treatment. Both the negative control (non-treated) and A2 treated fish epithelial cells showed no signs of apoptosis under microscopy for DHE staining (Figures 5(a) and (b)). HT-29 cells that were treated with PACs and stained with DHE fluoresced red when imaged under confocal microscopy. Sections of the cytosol appeared to illuminate at the expected wavelength during earlier time points of 1 - 2 hours, but then became concentrated in the nuclei as the 5 hour exposure time approached; Negative control cells did not fluoresce significantly under fluorescence (Figure 6). Analysis showed that the PACs treatment caused more apoptosis to occur (78.4\%) than in untreated control (Figure 7). ANOVA variance tests
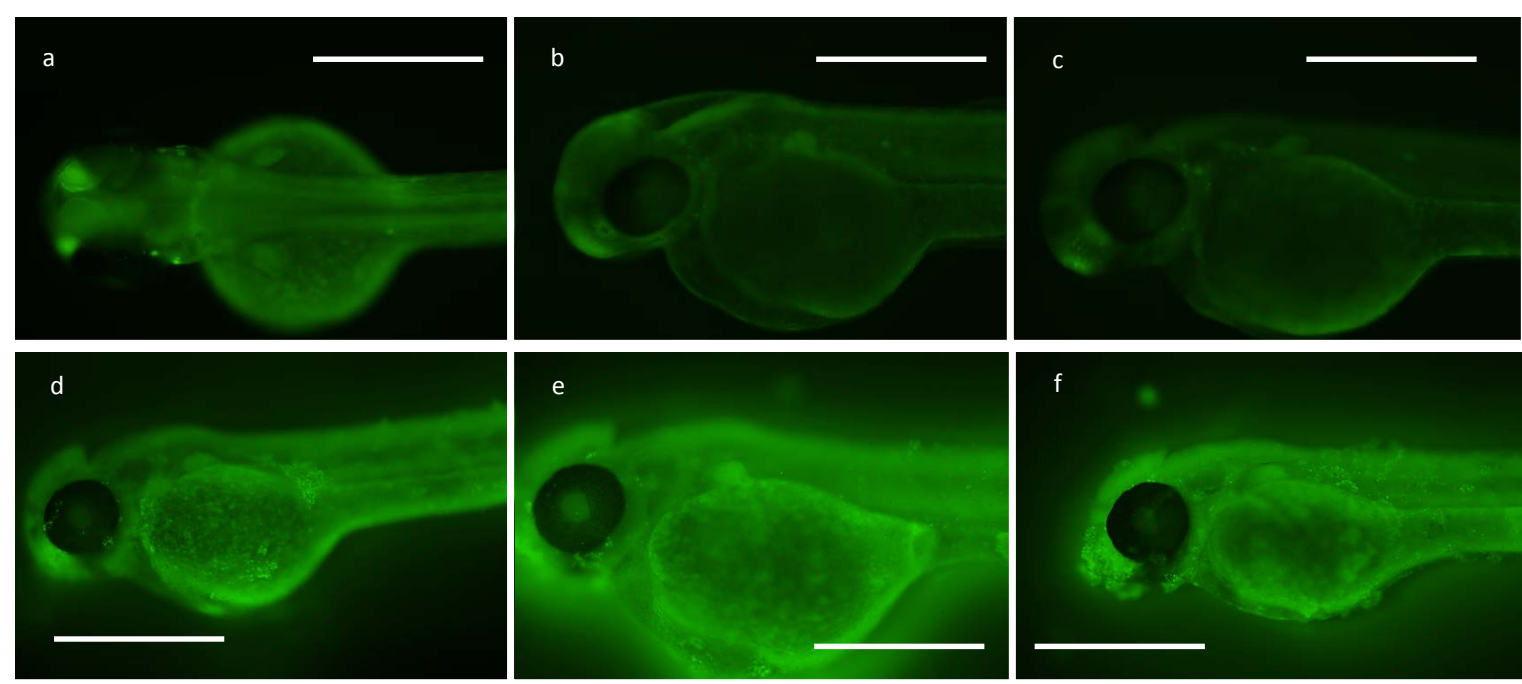

Figure 4. Acridine orange apoptosis screening for cell death. Bar $=500 \mu \mathrm{m}$. All PAC treated groups are in solution at $100 \mu \mathrm{g} / \mathrm{mL}$ concentration. (a) $48 \mathrm{hpf}$ in DMSO at $100 \mu \mathrm{g} / \mathrm{mL}$ control (5 hr); (b) $48 \mathrm{hpf}$ in $\mathrm{dH}_{2} \mathrm{O}$ control (2 hr, $35 \mathrm{~min}$ ); (c) 48 hpf in $\mathrm{dH}_{2} \mathrm{O}$ control (5 hr); (d) 48 hpf in PAC (1 hr); (e) 48 hpf in PAC (1 hr, 55 min); (f) 48 hpf in PAC (2 hr, $35 \mathrm{~min})$. 

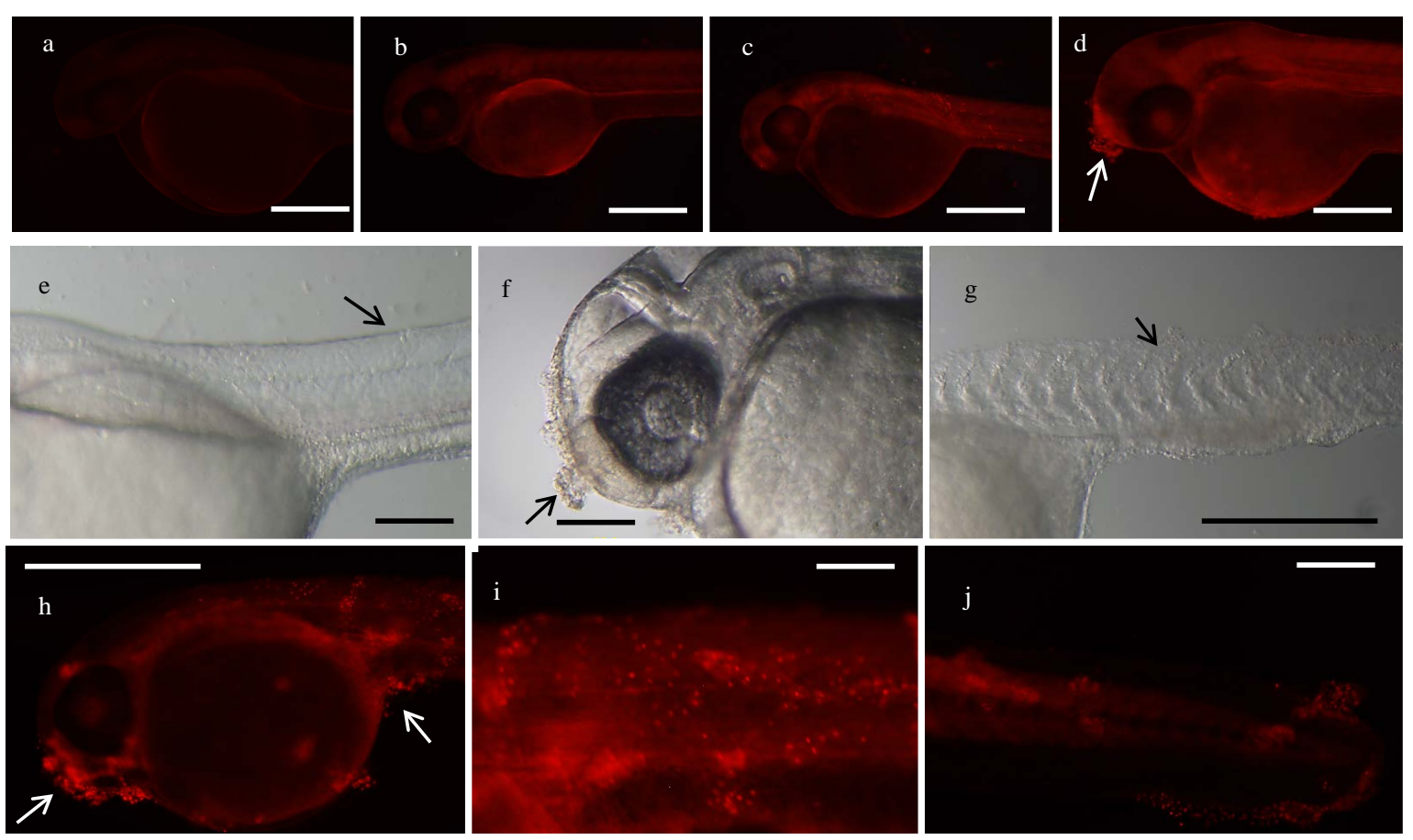

Figure 5. DHE staining for cellular superoxide presence in $48 \mathrm{hpf}$ fish. All treated groups are at $100 \mu \mathrm{g} / \mathrm{mL}$ concentration. (a) $\mathrm{dH}_{2} \mathrm{O}$ control (5 hr), bar = $200 \mu \mathrm{m}$; (b) A2 PAC (5 hr), bar = $500 \mu \mathrm{m}$; (c) smPAC (5 hr); (d) PAC (1 hr, $55 \mathrm{~min}$ ); (e) smPAC (5 hr), bar = $200 \mu \mathrm{m}$; (f): PAC (1 hr, $55 \mathrm{~min})$; (g) PAC (5 hr); (h) bar = $500 \mu \mathrm{m}$ : PAC (4 hr); (i) PAC (4 hr), bar $=200 \mu \mathrm{m}$; (j) PAC (2 hr, $35 \mathrm{~min})$, bar $=200 \mu \mathrm{m}$.
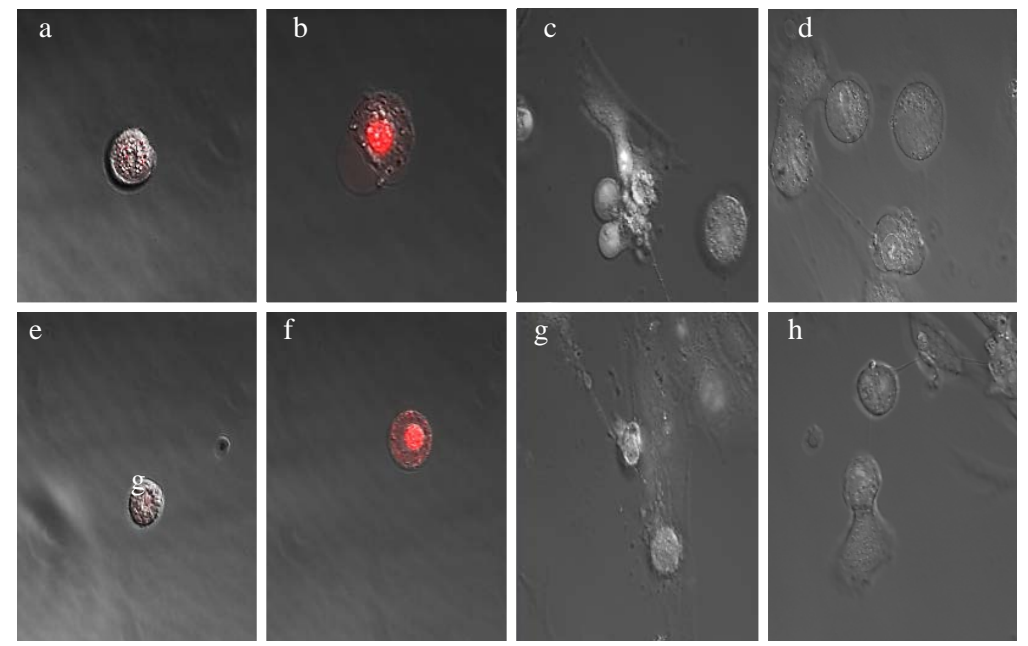

Figure 6. Scanning laser confocal images. (a), HT-29 untreated control (2 hr, 35 min); (b), HT-29 PAC treated (2 hr, 35 min); (c), CCD-CO18 untreated control (2 hr, $35 \mathrm{~min})$; (d), CCD-CO18 PAC treated (2 hr, $35 \mathrm{~min})$; (e), HT-29 untreated control (5 hr); (f), HT-29 PAC treated (5 hr); (g), CCD-CO18 untreated control $(5 \mathrm{hr})$; (h), CCD-CO18 PAC treated $(5 \mathrm{hr})$. DHE at $3 \mu \mathrm{M}$ was used to detect superoxide presence in cells under confocal microscopy. HT-29 cells were observed to develop red nuclei at the last two imaging time points, while CCD-CO18 cells did not fluoresce significantly.

confirmed that results were significant $\left(\mathrm{F}>\mathrm{F}_{\mathrm{CRIT}}\right.$ ). Normal CCD-CO18 colon cells treated with PACs were observed to be less apoptotic (48.67\%) when compared to HT-29 colon cells that were exposed to the same concentration. Further, unlike the HT-29 cells, the normal colon cell line did not fluoresce under confocal imaging 


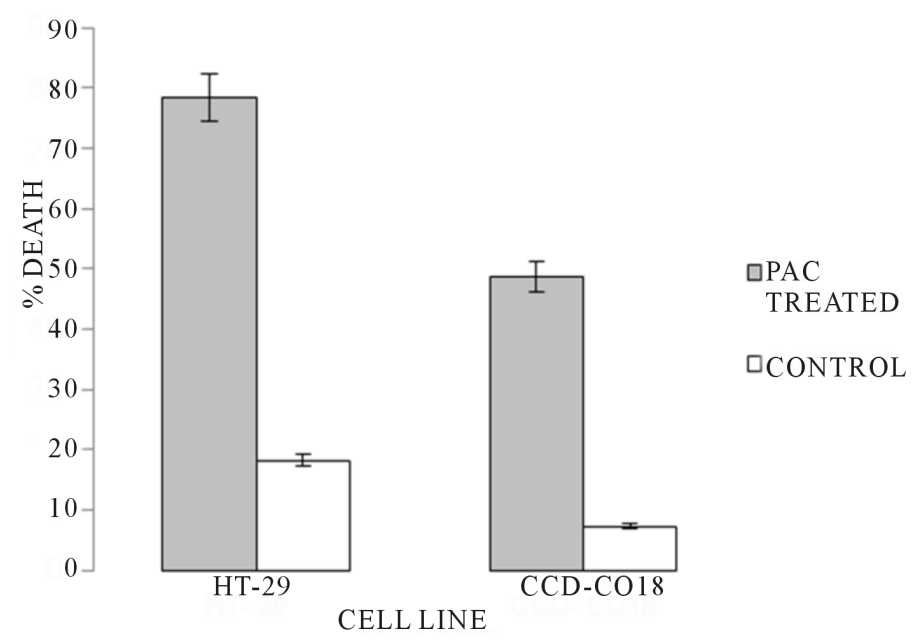

Figure 7. Trypan blue viability data. After a $5 \mathrm{hr}$ exposure period, cell viability of culture groups was tested. HT-29 PAC treated groups demonstrated significantly more death due to treatment (78.4\% death) when compared to non-treated controls (18.22\% death). The number of apoptotic CCD-CO18 treated cells (48.67\% death) was shown to be less than in HT-29 treated; however, based on CCD-CO18 control $(92.78 \%$ viable), treated non-cancerous cells still experienced lower level cytotoxicity in treatment.

of DHE for superoxide presence. ANOVA analysis confirmed that the cancer cells had significantly higher levels of apoptosis $\left(\mathrm{F}>\mathrm{F}_{\mathrm{CRIT}}\right)$.

\section{Conclusions}

One of the initial goals of this work was to test the feasibility of using the zebrafish model to examine tumor inhibition, to follow up on much in vitro work suggesting that cranberry PACs inhibit colon cancer cell proliferation (Liberty et al., in preparation). One challenge when evaluating compounds for efficacy in vivo is looking at metabolism and how those compounds circulate throughout the tissue and other bodily fluids. We therefore, set out to examine organ function in the zebrafish upon exposure to PACs. At $48 \mathrm{hpf}$ the liver is functioning as well as kidneys, however, examination of AHR2 and CYP3A65 expression did not indicate metabolic stress that could explain the death of the embryos when treated with PACs (Figure 3). The increase in expression of the genes across the last exposure time point is likely in response to organ system failure immediately prior to death. Furthermore, it is likely that the slight increase in CYP3A65 may be in response to the increase in AHR2 since CYP3A65 transcription has been shown to be induced by AHR activation [21].

While the PACs did not cause metabolic stress, there was clear damage to the surface epithelium of the embryos. Several stages of development were assayed with varying concentrations of PACs. Embryos ranging from 24 hpf up to $7 \mathrm{dpf}$ suffered loss of epithelial adherence properties upon treatment until eventual yolk sac bursting. PAC treatment in cell culture demonstrated that proanthocyanidins are more cytotoxic to cancerous epithelial cells (HT-29) than the non-cancerous type utilized in this study (CCD-CO18). This naturally led us to examine apoptosis in the zebrafish embryos.

Acridine orange apoptosis indicator allowed for pinpointing where exactly along the fish tissue that the cells were being affected by the PACs. In distilled water and DMSO controls, no apoptotic behavior was detected on $48 \mathrm{hpf}$ zebrafish cells at any time of exposure indicated by the absence of green fluorescence during microscopy observations (Figure 4). Only PACs-treated fish showed evidence of apoptotic cells under microscopy, and it was possible to identify specific areas along the zebrafish where cells were being affected by the treatment. Small sections of damaged tissue were located in disperse sections all around the yolks of the fish and spotted along the length of the tail, but the most concentrated site of apoptotic cells was around the urogenital pore region and the area at the mouth of the fish (Figure 4). As exposure to the treatment approached 5 hours, tissue of the $48 \mathrm{hpf}$ fish began to disassociate in large but fine flakes along with a shattered gut and yolk sac. When early 
embryos (1-cell to 6hpf) were exposed to PACs, we found that only the dechorionated embryos were affected, while the embryos within the chorion were unaffected (data not shown). This suggests that the chorion of the zebrafish acted as a protective barrier, and was selective to the PACs molecules in solution not allowing the compounds to come in contact with the cells of the developing embryo. One possibility is that the PACs may be acting through direct contact with the cell surface. The PACs may act as a ligand and bind to the surface of cells as the molecules come in contact with them having the inability to pass through the cell membrane due to the large size of the PAC molecule as a condensed tannin compound with molecular weights of up to $3000 \mathrm{amu}$.

Also, in finding that superoxides were detected in both cell culture and in vivo by DHE staining, it is possible that the PACs compounds bind to the surface of cells, triggering a pathway which leads to the disruption of membrane potential by mitochondrial failure thus releasing superoxides into the cytoplasm of cells which then penetrate the nucleus and damage DNA. A similar effect was seen when zebrafish embryos were exposed to the anti-cancer polypeptide pleurocidin [17].

Interestingly, when exposed to PACs, 2-month-old adult zebrafish seemed to develop a darkened color gut over the course of treatment eventually losing the ability to swim, and ultimately suffering heart failure. The lack of epithelial cell damage could be due to the increased toughness of outermost epithelium layer in the older fish tissue, while the inner intestinal epithelium layer could still have sustained tissue damage since the PACs were constantly being absorbed through the gills and ingested orally through the mouth of the fish. As stated, no change in $\mathrm{pH}$ of the treatment solution in water from an initial $\mathrm{pH}$ of 7.0 to either a significantly more basic or acidic medium was recorded so it can be said that significant acid or basic substances are not being generated during the assay which would have potentially resulted in disassociation of the fish tissue. The intestinal epithelium has a higher rate of proliferation than does the skin of the 2 month old fish which is consistent with cell death in embryonic zebrafish cells that have a higher rate of cell division, similar to cancer cells. The PACs have been shown to have an effect on the G2 phase of the cell cycle (Liberty et al., in preparation), and may be more damaging to rapidly proliferating cells. It is also well known that intestinal epithelial cells consistently are shed and undergo a form of apoptosis known as anoikis [23]. Anoikis is a natural loss of cell anchorage which is dependent on caspase activation and cytochrome $C$ release by the mitochondria [24]. Similar detachment is seen in the surface epithelium of the embryos when exposed to PACs. Anoikis is further supported by the dramatic decrease in COX2A expression during PACs exposure (Figure 3). COX2A has numerous roles in cancer cell growth, including inhibition of anoikis [25]. Decreased COX2A would relieve the inhibition of anoikis allowing it to occur more readily. When anoikis is inhibited cells do not die, rather they undergo epithelial-mesenchymal transition (EMT) which allows them to detach and re-attach instead of dying, the hallmark of metastasis. We suggest that the interaction of PACs at the cell surface promotes a signaling cascade that inhibts COX2A thereby promoting anoikis. Cancer cells require MAPk signaling to enter EMT and avoid anoikis. We also have preliminary data suggesting that PACs causes a decrease in MAPk expression (liberty et al., in preparation).

Future work will focus on examining the potential of PACs to promote anoikis and inhibit key molecules such as COX2A.

\section{Conflict of Interests}

The authors hereby declare that they have no conflicts of interest.

This research was funded by UMass Dartmouth cranberry research grant to Neto and Ferreira, and approved under IACUC protocol\# 13-01. This work was also funded in part by a College of Engineering Undergraduate Research Incentive Program (URIP) award to Nicholas Macedo.

All the authors of this paper have no financial relationship with the institution where the research was carried out.

\section{References}

[1] Dohadwala, M.M., Holbrook, M., Hamburg, N.M., Shenouda, S.M., Chung, W.B., Titas, M., Kluge, M.A., Wang, N., Palmisano, J., Milbury, P.E., Blumberg, J.B. and Vita, J.A. (2011) Effects of Cranberry Juice Consumption on Vascular Function in Patients with Coronary Artery Disease. The American Journal of Clinical Nutrition, 93, 934-940. http://dx.doi.org/10.3945/ajcn.110.004242

[2] Cesonienè, L., Jasutienè, I. and Šarkinas, A. (2009) Phenolics and Anthocyanins in Berries of European Cranberry and Their Antimicrobial Activity. Medicina (Kaunas), 45, 992-999. 
[3] Caillet, S., Côté, J., Sylvain, J.F. and Lacroix, M. (2012) Antimicrobial Effects of Fractions from Cranberry Products on the Growth of Seven Pathogenic Bacteria. Food Control, 23, 419-428.

http://dx.doi.org/10.1016/j.foodcont.2011.08.010

[4] Neto, C.C. (2007) Cranberry and Its Phytochemicals: A Review of in Vitro Anticancer Studies. The Journal of Nutrition, 137, 186S-193S.

[5] Kim, K.K., Singh, A.P., Singh, R.K., DeMartino, A., Brard, L., Vorsa, N., Lange, T.S. and Moore, R.G. (2012) AntiAngiogenic Activity of Cranberry Proanthocyanidins and Cytotoxic Properties in Ovarian Cancer Cells. International Journal of Oncology, 40, 227-235. http://dx.doi.org/10.3892/ijo.2011.1198

[6] Nandakumar, V., Singh, T. and Katiyar, S.K. (2008) Multi-Targeted Prevention and Therapy of Cancer by Proanthocyanidins. Cancer Letters, 269, 378-387. http://dx.doi.org/10.1016/j.canlet.2008.03.049

[7] Neto, C.C., Krueger, C.G., Lamoureaux, T.L., Kondo, M., Vaisberg, A.J., Hurta, R.A.R., Curtis, S., Matchett, M.D., Yeung, H., et al. (2006) MALDI-TOF MS Characterization of Proanthocyanidins from Cranberry Fruit (Vaccinium macrocarpon) That Inhibit Tumor Cell Growth and Matrix Metalloproteinase Expression in Vitro. Journal of the Science of Food and Agriculture, 86, 18-25. http://dx.doi.org/10.1002/jsfa.2347

[8] Yokota, K., Kimura, H., Ogawa, S. and Akihiro, T. (2013) Analysis of A-Type and B-Type Highly Polymeric Proanthocyanidins and Their Biological Activities as Nutraceuticals. Journal of Chemistry, 2013, Article ID: 352042. http://dx.doi.org/10.1155/2013/352042

[9] Kresty, L.A., Howell, A.B. and Baird, M. (2011) Cranberry Proanthocyanidins Mediate Growth Arrest of Lung Cancer Cells through Modulation of Gene Expression and Rapid Induction of Apoptosis. Molecules, 16, 2375-2390. http://dx.doi.org/10.3390/molecules16032375

[10] Howe, K., Clark, M.D., Torroja, C.F., Torrance, J., Berthelot, C., Muffato, M., Collins, J.E., et al. (2013) The Zebrafish Reference Genome Sequence and Its Relationship to the Human Genome. Nature, 496, 498-503 http://dx.doi.org/10.1038/nature12111

[11] Kim, D.-S., Huh, J.-W., Kim, Y.-H., Park, S.-J., Kim, H.-S. and Chang, K.-T. (2010) Bioinformatic Analysis of TESpliced New Exons within Human, Mouse and Zebrafish Genomes. Genomics, 96, 266-271. http://dx.doi.org/10.1016/j.ygeno.2010.08.004

[12] Santoriello, C. and Zon, L.I. (2012) Hooked! Modeling Human Disease in Zebrafish. JCI: The Journal of Clinical Investigation, 122, 2337-2343. http://dx.doi.org/10.1172/JCI60434

[13] Steffen, L.S., Guyon, J.R., Vogel, E.D., Beltre, R., Pusack, T.J., Zhou, Y., Zon, L.I. and Kunkel, L.M. (2007) Zebrafish Orthologs of Human Muscular Dystrophy Genes. BMC Genomics, 8, 79. http://dx.doi.org/10.1186/1471-2164-8-79

[14] Drummond, I.A. (2005) Kidney Development and Disease in the Zebrafish. JASN Journal of the American Society of Nephrology, 16, 299-304. http://dx.doi.org/10.1681/ASN.2004090754

[15] Field, H.A., Ober, E.A., Roeser, T. and Stainier, D.-Y.R. (2003) Formation of the Digestive System in Zebrafish. I. Liver Morphogenesis. Developmental Biology, 253, 279-290. http://dx.doi.org/10.1016/S0012-1606(02)00017-9

[16] Feitsma, H. and Cuppen, E. (2008) Zebrafish as a Cancer Model. The American Association for Cancer Research. Molecular Cancer Research, 6, 685-694. http://dx.doi.org/10.1158/1541-7786.MCR-07-2167

[17] Morash, M.G., Douglas, S.E., Robotham, A., Ridley, C.M., Gallant, J.W. and Soanes, K.H. (2011) The Zebra Fish Embryo as a Tool for Screening and Characterizing Pleurocidin Host-Defense Peptides as Anti-Cancer Agents. Disease Models \& Mechanics, 4, 622-633. http://dx.doi.org/10.1242/dmm.007310

[18] Patel, K.D., Scarano, F.J., Kondo, M., Hurta, R.A.R. and Neto, C.C. (2011) Proanthocyanidin-Rich Extracts from Cranberry Fruit (Vaccinium macrocarpon, Ait.) Selectively Inhibit the Growth of Human Pathogenic Fungi Candida spp. and Cryptococcus neoformans. Journal of Agricultural and Food Chemistry, 59, 12864-12873. http://dx.doi.org/10.1021/jf2035466

[19] Kent, M.L., Bishop-Stewart, J.K., Matthews, J.L. and Spitsbergen, J.M. (2002) Pseudocapillariatomentosa, a Nematode Pathogen, and Associated Neoplasms of Zebrafish (Daniorerio) Kept in Research Colonies. Comparative Medicine, 52, 354-358.

[20] Gu, L., Kelm, M.A., Hammerstone, J.F., Beecher, G., Holden, J., Haytowitz, D., Gebhardt, S. and Prior, R.L. (2004) Concentrations of Proanthocyanidins in Common Foods and Estimations of Normal Consumption. The Journal of Nutrition, 134, 613-617.

[21] Chang, C.T., Chung, H.Y., Su, H.T., Tseng, H.P., Tzou, W.S. and Hu, C.H. (2013) Regulation of Zebrafish CYP3A65 Transcription by AHR2. Toxicology and Applied Pharmacology, 270, 174-184. http://dx.doi.org/10.1016/j.taap.2013.04.010

[22] Silva, T.L., Cox, A.A., Boominathan, V.P., Jezewski, P.A. and Ferreira, T.L. (2012) Early Expression of the tbx22 Gene in Zebrafish Influences Positioning of Pharyngeal Arch Cartilages. American Journal of Molecular Biology, 2, 318-331. http://dx.doi.org/10.4236/ajmb.2012.24033 
[23] Frisch, S.M., Schaller, M. and Cieply, B. (2013) Mechanisms That Link the Oncogenic Epithelial-Mesenchymal Transition to Suppression of Anoikis. Journal of Cell Science, 126, 21-29. http://dx.doi.org/10.1242/jcs.120907

[24] Grossmann, J. (2002) Molecular Mechanisms of “Detachment-Induced Apoptosis-Anoikis”. Apoptosis, 7, $247-260$. http://dx.doi.org/10.1023/A:1015312119693

[25] Choi, E.M., Kwak, S.J., Kim, Y.M., et al. (2005) COX-2 Inhibits Anoikis by Activation of the PI-3K/Akt Pathway in Human Bladder Cancer Cells. Experimental and Molecular Medicine, 37, 199-203.

http://dx.doi.org/10.1038/emm.2005.27

\section{ABBREVIATION LIST}

EB: Early Black

PAC: Proanthocyanidins (DP < 10)

A2 PAC: Proanthocyanidin Dimer (alpha type)

smPAC: Proanthocyanidins (DP < 6)

DHE: Dihydroethidium

CCD-CO18: Non-cancerous Human Colon Epithelial Cell

HT-29: Cancerous Human Colon Epithelial Cell

Hpf: Hours Post Fertilization

PCR: Polymerase Chain Reaction 